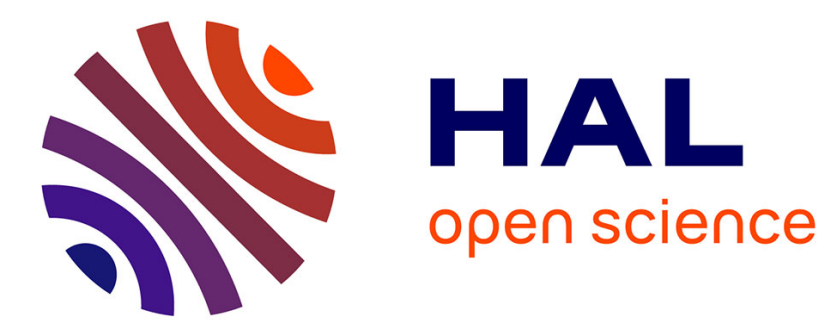

\title{
Ratchet-like motion of a shaken drop
}

\author{
Xavier Noblin, Richard Kofman, Franck Celestini
}

\section{To cite this version:}

Xavier Noblin, Richard Kofman, Franck Celestini. Ratchet-like motion of a shaken drop. Physical

Review Letters, 2009, 102 (19), pp.194504. hal-00455096

\section{HAL Id: hal-00455096 \\ https://hal.science/hal-00455096}

Submitted on 9 Feb 2010

HAL is a multi-disciplinary open access archive for the deposit and dissemination of scientific research documents, whether they are published or not. The documents may come from teaching and research institutions in France or abroad, or from public or private research centers.
L'archive ouverte pluridisciplinaire HAL, est destinée au dépôt et à la diffusion de documents scientifiques de niveau recherche, publiés ou non, émanant des établissements d'enseignement et de recherche français ou étrangers, des laboratoires publics ou privés. 


\title{
Ratchet-like motion of a shaken drop
}

\author{
Xavier Noblin, Richard Kofman, and Franck Celestini* \\ Laboratoire de Physique de la Matière Condensée ,UMR 6622, CNRS, \\ Université de Nice Sophia-Antipolis, Parc Valrose 06108 Nice Cedex 2, France
}

(Dated: March 23, 2009)

\begin{abstract}
We study the motion of a drop lying on a plate simultaneously submitted to horizontal and vertical harmonic vibrations. The two driving vibrations are adjusted to the same frequency, and according to their relative amplitude and phase difference $\Delta \Phi$, the drop experiences a controlled directed motion with a tunable velocity. We present a simple model putting in evidence the underlying mechanism leading to this ratchet-like motion of the drop. Our model includes the particular case $\Delta \Phi=\pi$ corresponding to the climbing of a drop on a vertically vibrated inclined substrate, as recently observed by Brunet et al. (Phys. Rev. Lett. 99, 144501 (2007)). This study gives insights in the fundamental issue of wetting dynamics and offers new possibilities of controlled motion in droplet microfluidics applications.
\end{abstract}

PACS numbers: 47.55.dr,68.35.Ja,68.08.Bc

Microfluidics has recently motivated both fundamental and applied researches [1]-[2]. The challenge is to find an optimal process to manipulate small liquid quantities, down to the nanoliter scale, in order to study chemical reactions, biological molecules and processes, or to perform biomedical tests in a rapid and repetitive way. Two main ways have emerged in order to handle drops: two-phase flows in microchannels [1]-[3] and sessile drops displacement on surfaces [4]-[6]. For the latter, the existence of a contact angle hysteresis prevents the motion of the drop. Nevertheless this difficulty can be overcome using drop oscillations modes induced by vibrations. More than a century ago, Rayleigh [7] and Kelvin [8] found a general expression for these modes due to surface tension, in the case of free liquid drops. In the 1980's, microgravity experiments have motivated studies on sessile drops oscillations [9] and several works have then focused on the forced vibrations of supported drops [10]-[12] for which the contact line is fixed. A drop behaves like a spring with different resonant frequencies at which a small vibration induces a significant deformation. Locally, the contact angle can become respectively higher or lower than the advancing $\left(\theta_{a}\right)$ or receding $\left(\theta_{r}\right)$ contact angles, the critical values of the wetting hysteresis inducing contact line motion.

Such a reduction of the effect of contact angle hysteresis $[13,14]$ to induce drop motion has been first illustrated by Daniel et al. [15]. They performed experiments on a drop deposited on a substrate with a continuous wettability gradient and demonstrated that parallel vibrations applied to the substrate helped the drop to move toward its more wettable part. These authors with de Gennes [16] also put in evidence the possibility to use asymmetric horizontal vibrations to control the drop motion without any external force. Vertical vibrations have also demonstrated their ability to overcome contact angle hysteresis effects [17], allowing contact line motion, but not a net drop motion. It comes out that horizontal or vertical symmetrical vibrations alone do not provide the ratchetlike asymmetry necessary to induce a net drop displacement. Brunet et al. [18] observed recently the surprising climbing of a drop on a vibrated inclined plane. This forced motion can be decomposed into parallel and perpendicular vibrations of the substrate, as being a particular case of the present study with a phase difference $\Delta \Phi=\pi$.

In this letter, we demonstrate that the combination of horizontal and vertical vibrations induces an asymmetry and a net drop motion. By simply tuning the phase difference between the two vibrations, a direction or another is chosen at a given controlled velocity. We put in evidence the unexpected rich relation between $\Delta \Phi$ and the average velocity of the drop $V$. We first describe the experimental apparatus used to combine horizontal and vertical vibrations in a way to reduce their possible coupling. The method used to obtain the relation between the phase difference and the drop velocity is then presented together with the results obtained for different horizontal and vertical vibrations. A simple analysis and a mechanical model are finally proposed to explain how the combination of vertically and horizontally excited modes leads to a net motion of the drop and the fundamental role of the phase difference $\Delta \Phi$.

The experimental apparatus depicted in Fig. 1. consists in two independent vibration exciters. The vibrations induce substrate motion in the horizontal $(\mathrm{h})$ and vertical (v) directions : $a_{h}(t)=A_{h} \cos \left(2 \pi f_{h} t\right)$ and $a_{v}(t)=A_{v} \cos \left(2 \pi f_{v} t+\Delta \Phi\right)$. The main difficulty has consisted in reducing the mechanical coupling between both vibrations. Our setup allowed to minimize this coupling to roughly $5 \%$. The experiments are performed under a laminar flow hood in order to reduce dust contamination of the substrate. A water drop of radius $1.3 \mathrm{~mm}$ is deposited on a cross-linked PDMS substrate (Sylgard 184). We measured a wetting angle of $96^{\circ}$ and an hysteresis $\theta_{a}-\theta_{r} \approx 19^{\circ}$. The drop has a first resonant frequency 

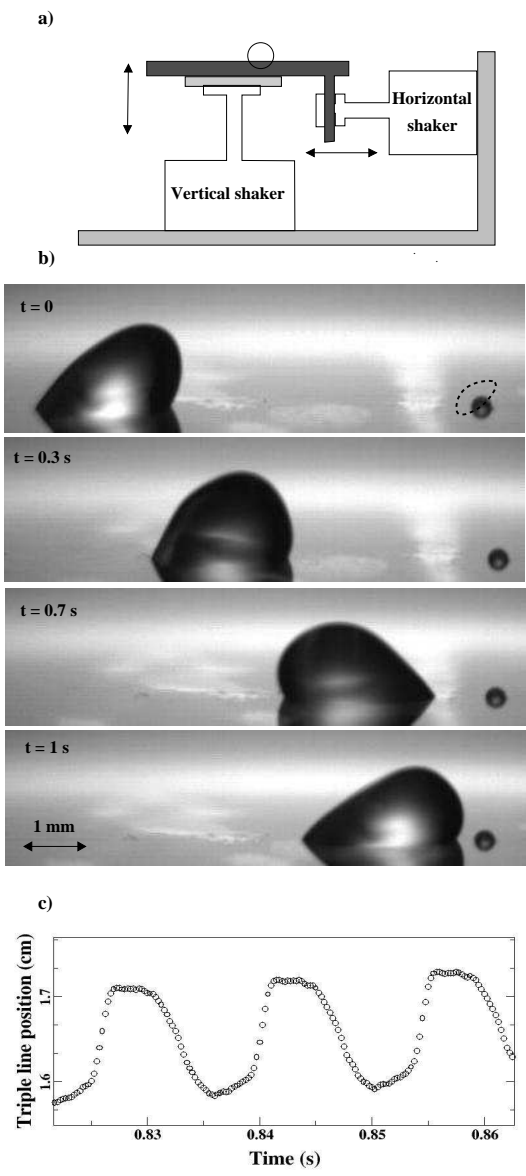

FIG. 1: a) Experimental setup used to combine vertical and horizontal vibrations of the substrate. b) Images taken from different vibrations cycles at various oscillation phases. Here $f_{v}=f_{h}=70 H z, \phi=\pi / 4 A_{v}=140 \mu \mathrm{m}$ and $\left.A_{h}=300 \mu \mathrm{m} . \mathrm{c}\right)$ Position of the triple line on the right versus time. The lines are guide to the eyes to put in evidence the stops of the triple line.

at $f_{h}^{0}=46 \mathrm{~Hz}$ corresponding to the "rocking mode" associated to parallel vibration of the substrate. This experimental value is in good agreement with the semianalytical expression proposed recently [19] and predicting a resonance at $43 \mathrm{~Hz}$. At $f_{v}^{0}=96 \mathrm{~Hz}$ the second resonant frequency corresponds to the "pumping mode" which is induced by perpendicular vibration of the surface. Even if the pumping mode is somehow related to the $n=2$ Lamb's mode, its value is different since the contact angle value of the supported drop strongly influences its resonance [9]-[12], [17], [20], [21].

In most of our experiments $f_{h}=f_{v}=f$, with a value just in between $f_{h}^{0}$ and $f_{v}^{0}$ that leads to the largest drop motion. For lower or higher frequencies, only the rocking or pumping modes are respectively excited and no motion is observed. We used a high-speed camera (Photron Ul- tima 512) to visualize the drop from the side, looking at its deformation and its trajectory at a frame rate ranging from 1000 to 8000 images per second. The pictures in Fig. $1 \mathrm{~b}$ represent the shapes of the drop at different phases of its motion, recorded during $1 \mathrm{~s}$ with $f=70 \mathrm{~Hz}$. The drop therefore experiences seventy cycles of oscillations. Different phases are taken from different cycles to make the overall displacement more visible. The average velocity is $0.68 \mathrm{cms}^{-1}$ which corresponds to a displacement of $8 \%$ of the drop radius during each vibration cycle. The dotted line is a Lissajous curve representing the trajectory of the small drop at rest on the side of the substrate. This figure leads to the measurement of $\Delta \Phi, A_{h}$ and $A_{v}$. The ratchet like motion of the drop is evidenced in the Fig. $1 \mathrm{c}$ representing the position of the triple line on the right recorded

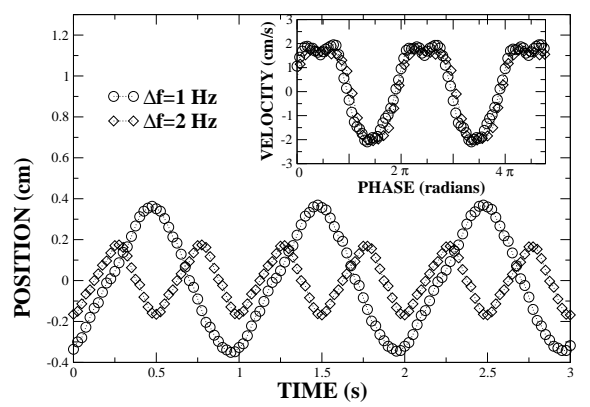

FIG. 2: Displacement of the droplet center of mass for two different frequency shifts $\Delta f=1$ and $2 \mathrm{~Hz}$. In inset: deducted drop velocity as a function of $\Delta \Phi$. The two data sets fall on the same curve.

Instead of making several measurements for each value of the phase difference, we determine the relationship between the drop velocity $V$ and $\Delta \Phi$ directly by applying two slightly different frequencies $f_{h}=f=70 \mathrm{~Hz}$ and $f_{v}=f+\delta f$. The phase is therefore time dependant, $\Delta \Phi(t)=2 \pi \delta f t$ and periodically takes all values between 0 and $2 \pi$. In Fig. 2, we represent the position of the center of mass of the drop as a function of time for two values of the frequency shift $\delta f=1$ and $2 H z$. As expected, since the phase is varying, the drop experiences a periodic motion on the substrate. The amplitude of this motion decreases with the frequency shift. For each curve we have extracted the drop velocity as a function of $\Delta \Phi$. This is represented in inset of the Fig. $2:$ the two data sets collapse on the same curve, validating our measurement procedure.

In Fig. 3a, we plot the velocity as a function of $\Delta \Phi$ for different values of $A_{h}$. The amplitude of vibration in the perpendicular direction is fixed to $A_{v}=140 \mu \mathrm{m}$. As function of $\Delta \Phi$ the drop undergoes a motion to the right $(V>0)$ or to the left $(V<0)$. We illustrate here that such combined parallel and perpendicular vibrations allow to control of the motion of the drop. We verify that the function $V(\Delta \Phi)$ satisfy $V(\Delta \Phi+\pi)=-V(\Delta \Phi)$, 

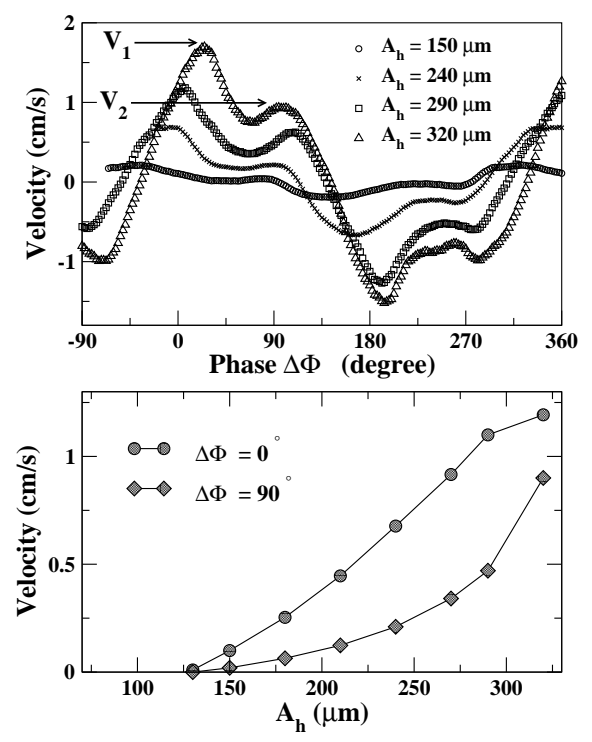

FIG. 3: a) Droplet velocities as a function of the phase difference for various parallel vibration amplitudes $A_{h}$. In inset, for a weak parallel vibration $A_{h}=130 \mu \mathrm{m}$ the two maxima are clearly visible. b) Velocity as a function of the $A h$.

small deviations being due to the presence of defects on the substrate. As expected, the velocity maximum $V_{1}$ depends on the amplitude of vibrations. We can also observe the existence of a second relative maximum $V_{2}$ in the $V(\Delta \Phi)$ curve. It is smaller than $V_{1}$ but nevertheless clearly visible whatever the value of $A_{h}$. For a weak vibration amplitude, the velocity is zero except around both maxima $V_{1}$ and $V_{2}$. In Fig. $3 \mathrm{~b}$, we plot $V$ as a function of $A_{h}$ for $\Delta \Phi=0$ and $90^{\circ}$. Below a threshold in amplitude the drop is at rest on substrate.

These observations can be understood by looking at the effect of vibrations on the drop dynamics. Consider a drop with a forced horizontal vibration. In that case, the drop presents the rocking mode corresponding to high value of the contact angle $\theta_{H}$ on a side and low value $\theta_{L}$ on the other side. When $\theta_{H}>\theta_{a}$ and $\theta_{L}<\theta_{r}$, the two sides of the drop start to move in the same direction inducing drop motion in the direction of the force. When the oscillation goes on, the drop moves back in the other direction so that the net motion over one period is null. Nevertheless, if at the same time a vertical vibration is imposed when the drop is deformed by the rocking mode, the deformation is different wether the vertical force is upward or downward. This leads to different variations in the contact angle, and nonsymmetric driving forces on the contact line between the right and left phases of the rocking mode. Finally, the motions to the left and to the right do not compensate each other. At each cycle the drop is displaced by the same amount in one direction, leading to a net motion. Depending on the phase difference between the two modes, the velocity will be positive, negative or null. We present a minimal model aiming at a better understanding of the $V$ versus $\Delta \Phi$ relationship. As depicted in Fig. 4a, a mass $m$ is attached to two vertical and two horizontal springs of respectively stifnesses $m \omega_{V}^{2} / 2$ and $m \omega_{h}^{2} / 2$. The springs are attached to a box deposited on an horizontal plane and submitted to vertical and horizontal vibrations. We denote $x_{1}$ the position of the box in the laboratory reference frame and $x$ and $y$ the mass coordinates in the box reference frame. We can write the following differential equations for $x$ and $y$ :

$$
\begin{aligned}
& \ddot{x}(t)=-A_{h} \omega^{2} \cos (\omega t)-\omega_{h}^{2} x-\alpha \dot{x} \\
& \ddot{y}(t)=-A_{v} \omega^{2} \cos (\omega t+\Delta \Phi)-\omega_{v}^{2} y-\alpha \dot{y}
\end{aligned}
$$

$\alpha$ is the dissipation coefficient associated to the mass motion in the box, representing internal dissipation in the fluid. The amplitudes $A_{h}$ and $A_{v}$ are due to the plane vibrations and $\Delta \Phi$ is the phase difference between these two excitations. We assume a solid friction between the box and the plane that gives rise to a large energy dissipation. Neglecting the box inertia, we can therefore write :

$$
\begin{array}{ll}
\dot{x}_{1}(t)=\Gamma_{1}\left(x-x_{c}\right) & \text { if } x>x_{c} \\
\dot{x}_{1}(t)=\quad 0 & \text { if }|x|<x_{c} \\
\dot{x}_{1}(t)=\Gamma_{1}\left(x+x_{c}\right) & \text { if } x<-x_{c}
\end{array}
$$

$\Gamma_{1}$ is the mobility of the box and $x_{c}$ is the displacement threshold value above which the box starts to move. It corresponds to a force through the spring stiffness, $\omega_{h}^{2} x_{c}$. Above this critical force in the $x$ direction, the friction does no longer completely compensate the driving force. We assume that $x_{c}=a+b y, a$ being a constant related to the load on the plane and $b y$ its modulation due to the vertical mass displacement. This mechanical system is a simple model of a drop shaken in the perpendicular and parallel directions. The two springs model the rocking and pumping modes of the drop. The solid friction mimics the effect of the wetting angle hysteresis. A similar analogy [16] has been successfully proposed, and latter numerically solved [22], to understand the motion of a drop under the action of non symmetrical vibrations.

We numerically solve this set of differential equations with the following parameters : $\omega_{h}=1, \omega_{v}=2, \omega=1.5$, $\alpha=\Gamma_{1}=a=b=1, A_{v}=0.5$ and we vary $A_{h}$ in between 1 and 1.5. As seen on Fig $4 \mathrm{~b}$ the box undergoes a ratchet-like motion qualitatively similar to the one observed experimentaly. The box velocity is represented in Fig4c as a function of $\Delta \Phi$ and in Fig4d as a function of $A_{h}$. The numerical results compare qualitatively well with the experiments represented in Fig 3 : We recover the same trend for $V(\Phi)$ as well as for $V\left(A_{h}\right)$. The asymmetry required for a net motion is introduced through the relation $x_{c}=a+b y$. For a real drop, $a$ is linked to the contact angle hysteresis, $x$ to the lateral motion induced 

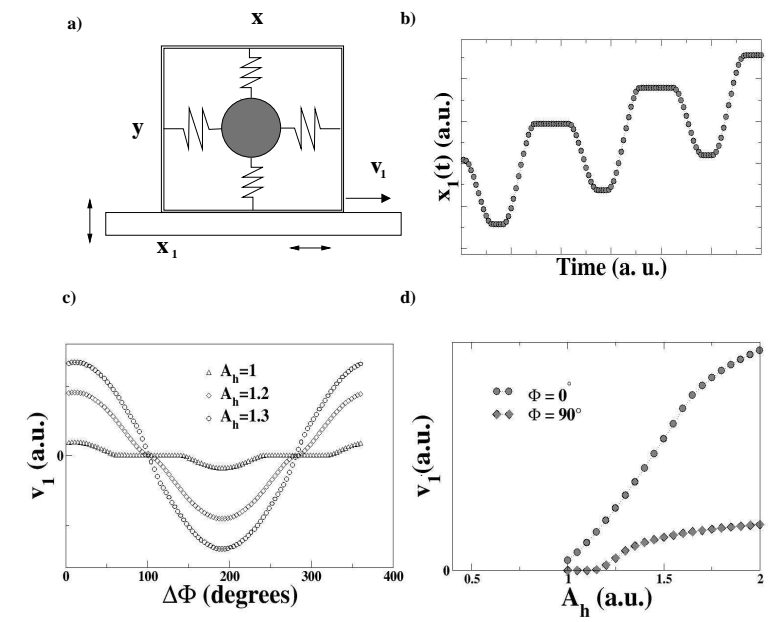

FIG. 4: a) Mechanical model. b) Position of the box as a function of time. c) Box velocity as a function of $\Delta \Phi$. d) Box velocity as a function of $A_{h}$.

by the rocking mode and $b y$ to the modulation associated to the pumping mode. The minimal assumptions used do not allow to observe a second maximum in the numerical $V(\Phi)$ while it clearly appears in experiments. This unexpected behavior should be due to nonlinearities of the drop oscillations and to a more complex force-velocity relationship that are not taken into account in our model.

In [18], Brunet et al. have observed the upward motion of a drop on an inclined substrate vertically vibrated. Our present study provides a direct explanation for their observations. Considering an inclined substrate presenting an angle $\theta$ with the horizontal plane, a drop is deposited and static due to the contact angle hysteresis. A vertical acceleration is induced on the substrate. By projecting it over the parallel and perpendicular directions of the plane, this corresponds to our system with $\Delta \Phi=\pi, \tan (\theta)$ giving the ratio between our "horizontal" and "vertical" vibration amplitudes. As it can be seen on our curves, $V(\pi)$ corresponds to a highly negative velocity, hence a climbing drop. The difference is that the driving force has to overcome gravity and that the drop is vibrated around an already asymmetrical-shape due to gravity, but this does not change the qualitative analysis. Finally we would like to point out the conection between our study and the one of Linke [23] et al on the selfpropelled motion of a Leidenfrost droplet. In that case the motion is due to the combined effects of the droplet pumping and to the asymetry substrate morphology presenting inclined slopes as for Brunet et. al.

To summarize, we have studied the effects of combined horizontal and vertical vibrations on a sessile drop. By varying the phase and relative amplitude between both vibrations, a net motion of the drop is possible. The corresponding average velocities have been measured as function of $\Delta \Phi$ and vibration amplitudes. At each vibra- tions cycle, a drop advances as much as $10 \%$ of its radius, leading to velocities of the order of $1 \mathrm{~cm} / \mathrm{s}$. This study leads to a better understanding of drops dynamics under vibrations and offers new possibilities to move them on a substrate. This could find applications in the dropletbased fluidics domain. Further studies are needed to understand the appearance of a second maximum for $V(\Phi)$. We plan to perform fluid dynamic simulations as recently done by Dong et al. [24] to understand the ratchet motion of a drop submitted to asymetric vibrations. These simulations should be useful to investigate the effect of the contact line force-velocity relation as well as nonlinearities in supported drop oscillations.

* Electronic address: Franck.Celestini@unice.fr

[1] Stone HA, Stroock AD, Ajdari A, Ann. Rev. of Fluid Mechanics, 36, 381-411 (2004)

[2] T. M. Squires and S. R. Quake, Rev. Mod. Phys.77, 977 (2005).

[3] H. Song, D.L. Chen, R.F. Ismagilov, Angewandte Chemie-International Edition, 45, 7336-7356 (2006).

[4] Pollack MG, Shenderov AD, Fair RB, Lab on a Chip. 2, 96 (2002).

[5] Cho SK, Moon HJ, Kim CJ, Jour. of Micromechanical. Syst. 12, 70 (2003).

[6] A.A. Darhuber and S.M.T. Troian, Ann. Rev. of Fluid Mechanics. 37, 425 (2005).

[7] Rayleigh, J. W. S., Proc. R. Soc. Lond. 29, 71 (1879).

[8] Lord Kelvin, Math. and Phys. papers, 3, 384 (1890).

[9] Bisch C, Lasek A, Rodot H, Jour. de Méca. Théo. et Appl. 1, 165 (1982)

[10] M. Strani and F. Sabetta, J. Fluid mech. 141, 233 (1984).

[11] Smithwick RW, Hembree DM, Jour. of Coll. and Int. Sci. 140, 57 (1990).

[12] Wilkes ED, Basaran OA, Phys. of Fluids. 9, 1512 (1997).

[13] E.L. Decker and S. Garoff, Langmuir 12, 2100 (1996).

[14] C. Andrieu, C. Sykes and F. Brochard, Langmuir 10, 2077 (1994).

[15] S. Daniel and M. K. Chaudhury, Langmuir 18, 3404 (2002).

[16] S. Daniel, M. K. Chaudhury and P. G. de Gennes, Langmuir 21, 4240 (2005).

[17] X. Noblin,A. Buguin and F. Brochart-Wyart, EPJE 14, 395 (2004).

[18] P. Brunet, J. Eggers and R. D. Deegan Phys. Rev. Lett 99, 144501 (2007).

[19] F. Celestini and R. Kofman, Phys. Rev. E 73, 41602 (2006).

[20] S. M. M. Ramos, Nucl. Instr. and Meth in Phys. Res. B 266, 3143 (2008).

[21] E. Bormashenko, R. Pogreb, G. Whyman, Y Bormashenko and M. Erlich. Appl. Phys. Lett. 90, 201917 (2007).

[22] D. Fleishman, Y. Asscher and M. Urbakh. J. Phys.: Condens. Matter 19, 96004 (2007).

[23] H. Linke, B. J. Aleman, L. D. Melling, M. J. Taormina, M. J. Francis, C. C. Dow-Hygelund, V. Narayan, R. P. Taylor and A. Stout. Phys. Rev. Lett 96, 154502 (2006).

[24] L. Dong, A. Chaudhury and M. K. Chaudhury. EPJE 
21, 231 (2006). 